\title{
Autour du darwinisme littéraire
}

\author{
Marc Lapprand \\ Université de Victoria
}

Raconter des histoires, inventer des fictions, créer des intrigues, en bref résider dans des mondes imaginaires sont autant de spécificités humaines sur lesquelles on s'interroge au moins depuis Aristote et Platon. Mais depuis une trentaine d'années, ces questions sont envisagées de plus en plus dans le cadre de l'évolution. Il s'agit de démontrer que la propension typiquement humaine à raconter et à consommer des histoires n'est pas seulement le produit dérivé d'un cerveau hypertrophié, mais répond à des besoins neurocognitifs engendrés par l'espèce. En d'autres termes, les narrations et, par extension, toutes les formes purement artistiques (non utilitaires) sont non seulement le résultat de notre tendance 
évolutive, mais tout simplement nécessaires à notre survie d'un point de vue adaptatif.

Selon Terrence Deacon, nous appartenons bel et bien à «l'espèce symbolique ». Le cerveau humain se caractérise, comme celui des primates, par une préfrontalisation importante. Le langage s'est ainsi peu à peu développé de sorte que notre cerveau s'est adapté à des besoins neurocognitifs croissants, en rapport direct avec notre motricité bipède et l'usage de nos bras et mains. Bref, ce n'est pas notre cerveau qui a évolué pour nous permettre d'y caser le langage, mais le contraire : c'est le cerveau qui s'est adapté à notre besoin vital de langage, comme un instinct (selon la théorie de Steven Pinker). C'est la symbolisation progressive chez l'humain qui lui a donné ce cerveau et le langage pour la véhiculer et la transmettre. Nous sommes donc mus par notre soif de représentation symbolique ${ }^{1}$ et, par extension, de raconter des histoires.

L'évolutionnisme ne fait pas consensus dans les milieux intellectuels, tant s'en faut. Pourtant, depuis l'ouvrage synthétique de Brian Boyd, On the Origin of Stories (2009), le temps est venu de se poser des questions concrètes sur ce que peut apporter cette nouvelle discipline dans le domaine des sciences humaines. C'est la raison principale qui a motivé ce dossier autour du darwinisme littéraire, fruit d'une journée de recherche tenue dans le cadre du congrès de l'APFUCC à l'Université de Victoria le 4 juin 2013. La lecture évolutionniste de la littérature n'est-elle qu'une autre forme de critique

1 Terrence Deacon, The Symbolic Species, New York, Londres, W. W. Norton \& Company, 1997. 
historique, comme l'avancent certains de ses détracteurs ?2 $\mathrm{Ou}$ alors peut-elle véritablement bouleverser nos concepts critiques solidement ancrés dans le structuralisme et ses disciplines héritières ? Qu'est-ce qui explique le foisonnement de ces recherches dans le monde anglo-saxon (Jonathan Gottschall, John Tooby, Leda Cosmides, Joseph Carroll, pour ne nommer qu'eux) et la relative frilosité du milieu intellectuel francophone à son égard? Cette science serait-elle victime de son apparente simplicité ? Les champs du savoir doivent-ils rester compartimentés, en vertu d'un certain constructivisme social qui a dominé le monde intellectuel pendant la seconde moitié du siècle dernier ? Ces questions ne sont évidemment pas exhaustives. Elles offrent un balayage très général des enjeux de cette épistémologie en formation, qui n'a pas encore atteint un niveau de maturité méthodologique ou théorique à proprement parler. Pourtant, vu ses avancées récentes, tout porte à croire que l'évolutionnisme est en train de s'implanter comme un nouveau champ de la connaissance et que, dès lors, on ne peut plus l'ignorer.

Alexandre Gefen le montre de manière convaincante : les enjeux épistémologiques diffèrent entre la critique anglosaxonne, qui amorce un retour inexorable vers la nature humaine, non pour expliquer la littérature, mais pour la poser biologiquement comme une donnée cognitive inévitable, tandis que la critique de tradition française reste encore largement fondée sur l'explication linguistique et sémiotique. Le clivage entre ces deux visions de l'objet littéraire est d'autant exacerbé par la montée de nos connaissances en neuro-cognitivisme, qui

\footnotetext{
2 Notamment dans Nicholas Saul et Simon J. James (dir.), The Evolution of Literature: Legacies of Darwin in European Cultures, Amsterdam, New York, Rodopi, 2011.
} 
à long terme nous permettront probablement d'accéder à un savoir, sinon total, du moins beaucoup plus approfondi, du fonctionnement réel du cerveau humain. C'est à partir de telles bases que Gottschall propose de parler des «nouvelles humanités » (new humanities).

Les trois articles suivants s'appliquent à montrer, par le biais d'études davantage ponctuelles, comment le darwinisme peut effectivement, voire efficacement, informer le littéraire. Marc Lapprand s'interroge sur la possibilité d'une méthodologie universelle, apte à rendre éventuellement compte de n'importe quel texte littéraire. Il propose en exemple le canon, Jane Austen, et, en contraste, un cas beaucoup plus récent et encore peu étudié, Martin Winckler, écrivain dont la formation de médecin et de gynécologue n'est pas sans répercussions directes dans son œuvre romanesque. Emilie Etemad envisage pour sa part «l'intertextualité sous le prisme de la théorie de l'évolution", fondant son analyse sur la Bibliothèque de Babel telle qu'elle est présentée par Borges. Stéphanie Posthumus, enfin, analyse La Possibilité d'une île de Michel Houellebecq, roman dans lequel, justement, la nature humaine et le monde post-humain sont en éternel conflit, à moins que les lois de l'évolution ne le régissent encore et toujours.

Martin Winckler nous offre, pour clore ce dossier, sa propre incursion dans le darwinisme littéraire, qui l'a séduit au point de nourrir à présent sa fiction. En primeur, et nous lui en savons gré, il nous en donne des extraits, commentés à la loupe de l'évolution, avec au passage des interprétations adaptatives de deux œuvres aussi diverses que Casablanca (le film) et Cyrano de Bergerac (la pièce). 
Le darwinisme littéraire a-t-il de beaux jours devant lui ? Il n'offre pas tant de solutions à une compréhension métacognitive du monde qu'il ne soulève des questions qui sont en passe de devenir de plus en plus « incontournables » dans le monde universitaire, particulièrement chez les chercheurs en sciences humaines. En d'autres termes, on peut avancer à bon droit que les grands principes structuralistes et poststructuralistes qui ont dominé la seconde moitié du siècle dernier auraient atteint leurs limites. Si le vingtième siècle aura été formaliste à bien des égards, et il fallait passer par cette importante étape du décryptage scientifique de l'objet littéraire, on peut augurer que ce siècle sera dominé par les recherches en sciences du comportement et du fonctionnement du cerveau humain et que si les chercheurs en neuroscience s'attachent essentiellement à une fonctionnalité biogénétique qui caractérise notre espèce, les retombées dans les champs littéraires et artistiques promettent d'être riches et stimulantes. Nous espérons que ce modeste dossier contribuera à faire avancer les choses dans ce sens. 